\title{
Technical aspects of trans reduction in margarines
}

\section{Technical aspects of trans reduction in margarines}

Oléagineux, Corps Gras, Lipides. Volume 7, Numéro 1, 95-8, Janvier - Février 2000, Dossier : actes des Journées Chevreul "Corps gras, nutrition et santé, questions d'actualité" (Bordeaux, Pessac)

Auteur(s) : Gerrit VAN DUIJN, TUSCC/Unilever Research, Olivier van Noortlaan 120, 3133 AT Vlaardingen, The Netherlands.

Author(s) : Gerrit VAN DUIJN

Résumé : The opinion of nutritional science on the effect of trans fatty acids on blood cholesterol has drastically changed during the last decade. As a reaction to these new findings, the European margarine industry decided in the mid nineties to eliminate trans containing components from their margarine fat phase compositions. This excluded practically the use of partially hydrogenated oils and fats. Trans-free margarines have been introduced with optimised fat crystal structures stabilising a maximum of water in oil emulsion with a minimum of solid fat phase. These fat crystal structures are formed by fat phase components obtained from interesterification and/or fractionation of nonhydrogenated and/or fully hydrogenated feedstocks.

Summary : The opinion of nutritional science on the effect of trans fatty acids on blood cholesterol has drastically changed during the last decade. As a reaction to these new findings, the European margarine industry decided in the mid nineties to eliminate trans containing components from their margarine fat phase compositions. This excluded practically the use of partially hydrogenated oils and fats. Trans-free margarines have been introduced with optimised fat crystal structures stabilising a maximum of water in oil emulsion with a minimum of solid fat phase. These fat crystal structures are formed by fat phase components obtained from interesterification and/or fractionation of nonhydrogenated and/or fully hydrogenated feedstocks.

Keywords : margarine, trans-fatty acids, hydrogenation, interesterification.

\section{ARTICLE}

\section{Introduction}

Prior to 1990, the reviews and studies on the nutritional and biological effects of trans fatty acids indicated that the effect of trans unsaturated fatty acids on blood cholesterol was comparable to that of cis unsaturated fatty acids. Both cis and trans fatty acids were considered to be better than saturated fatty acids. This opinion stimulated the use of trans instead of saturated fatty acids to give consistency to margarines.

Nutritional research, published since the early nineties [1, 2], indicates that the effect of trans fatty acids on blood cholesterol (and hence on coronary heart disease risk) is at least as adverse as that of saturated fatty acids. As a reaction to these new findings, Unilever decided in 1995 to practically 
eliminate trans containing components from their margarine fat phase compositions. Most European margarine manufacturers followed this decision. This trans reduction has been achieved without increasing the total level of blood cholesterol raising fats, while also the margarine properties remained unchanged.

The latest nutritional research shows an increasing evidence that trans fatty acids are probably worse than saturated fatty acids [3]. In November 1999, the FDA published the petition on compulsory labelling of trans fat next to saturated fats in the USA.

\section{Oils and fats for margarine production}

Margarines consist of an emulsion of small water droplets in oil stabilised by a structure of solid fat crystals (figure 1). The characteristics of the margarines are highly influenced by the properties of these solid fat crystals (specially their melting performance). The production of a large variety of margarines with properties optimised to the consumer's needs requires the availability of solid fats with a specific range of melting performances (the grey coloured area in figure 1).

The melting performance of margarines is normally expressed by the solid phase line. This gives the concentration of solids (determined by Nuclear Magnetic Resonance) as a function of temperature. For margarines, the temperature range from around $10^{\circ} \mathrm{C}$ (leaving the refrigerator) to around $40 \circ \mathrm{C}$ (melting in the mouth) is of importance.

Most of the oils and fats, naturally occurring in seeds (like soybean, sunflower, rapeseed, etc.), are too low in solids to give consistency to margarine.

Tropical oils like palm oil, palm kernel oil and coconut oil are higher in solids although their solid phase lines are still outside the optimal fat crystal characteristics area (figure 1). Structuring margarines with tropical oils would result in a high level of these oils in the blend to compensate for their lower effectiveness in margarine structuring. This has an unfavourable effect on blood cholesterol since tropical oils are high in saturated fatty acids (coconut oil around $90 \%$, palmkernel oil around $80 \%)$.

Modification of the solid phase lines of naturally occurring oils and fats is required to produce optimal solid fat phase components. These solid phase lines can be changed by three modification techniques:

- partial or full hydrogenation;

- interesterification;

- fractionation.

\section{Fat phase components with high trans levels}

Trans formation

Until the mid nineties, partial hydrogenation has been the most applied technique to increase the melting point of unsaturated oils and fats. 
Hydrogenation involves the addition of a hydrogen molecule across the unsaturated $\mathrm{C}=\mathrm{C}$ bonds in the fatty acid groups of the triacylglycerols, producing a more saturated fat. In the hydrogenation process, hydrogen gas is reacting with oil at elevated temperatures and pressures in the presence of a catalyst (normally Nickel on carrier). Products with different degrees of saturation can be obtained by stopping the reaction when only a part of the double bonds has reacted with hydrogen (partial hydrogenation).

Both positional and geometric (trans) isomers are formed to some extent during partial hydrogenation. These isomerisation reactions are promoted by a relative shortage of hydrogen at the catalyst surface. This shortage can be the result of process conditions (like hydrogen pressure, stirring energy and temperature) and/or by poisoning of the catalyst surface by sulphur.

Figure 2 shows the change of the trans fatty acid levels during the hydrogenation of Soybean oil at two different temperatures using a fresh and a partly sulphur poisoned catalyst. During the course of the reaction, the trans level rises until a maximum is reached. In thermodynamic equilibrium, two third of the double bonds will be in the trans-configuration and one third in the cis-configuration, hence, the maximum obtainable trans-level will be $67 \%$. In the shown example, this maximum is reached at a degree of saturation of around 30\% during the hydrogenation at high temperature using the partly sulphur poisoned catalyst. At high temperature with the fresh (non sulphur poisoned) catalyst the maximum trans-level is $42 \%$ reached at around $40 \%$ saturation and at low temperature $(120 \circ \mathrm{C})$ with fresh catalyst only $21 \%$ at around $50 \%$ saturation.

After this maximum the trans-level drops as both cis and trans unsaturated fatty acids are hydrogenated to saturated fatty acids. At full saturation the trans-level will be zero.

The lowest curve shown in figure 2 represents the minimum trans-levels obtainable under industrial conditions with the currently available Nickel based catalysts.

\section{Partially hydrogenated products}

Figure 3 shows the following development of the solid phase line of hydrogenated soybean oil at increasing degree of saturation:

- non hydrogenated soybean oil has no solids in the shown temperature range;

- line 1 shows the solids of soybean oil at $27 \%$ saturation, hydrogenated at a low temperature with a fresh catalyst (corresponding to the lowest curve of figure 2). These products were mainly used as stable frying oils;

- line 2 gives the solids at $46 \%$ saturation, hydrogenated at a high temperature with a fresh catalyst (corresponding to the middle curve of figure 2);

- line 3 is obtained at equal saturation and hydrogenation temperature as c) but by using a partly sulphur poisoned catalyst (trans according the highest curve of figure 2). The higher trans level results in increased solids concentrations at the lower temperatures ( 20 and $30 \circ \mathrm{C}$ ) but it does not 
contribute to the solids levels at the higher temperatures ( $>40^{\circ}$ ). The solid phase line will be steeper, which normally gives a positive contribution to the margarine characteristics;

- further saturation to 50 and $54 \%$ will increase the solids to the levels of lines 4 and 5 (trans according the highest curve of figure 2).

The above example illustrates that products with a wide range of melting performances can be produced by partial hydrogenation of soybean oil. Similar results can be obtained using different liquid oils and tropical oils as feedstocks.

The desired characteristics of partially hydrogenated fat phase components can be obtained by controlling the degree of saturation; optimising the process conditions like hydrogen pressure and oil temperature; the choice of the catalyst; and the selection of the oil/fat to be hydrogenated.

This made partial hydrogenation a very flexible tool to produce optimal cost-effective margarine fat phase components choosing from a range of available feedstocks.

\section{Very low trans alternatives}

\section{Trans-free margarines}

The effort to eliminate trans fatty acids in margarine strongly reduced or even eliminated the use of (trans-containing) partially hydrogenated oils/fats as solid fat phase components in margarines. Furthermore, the positive effect of the trans-content reduction on blood cholesterol should as little as possible be reduced by an increase of the saturated fatty acids level. This has been achieved by combination of:

- the production of margarines with optimised fat crystal structures to stabilise a maximum of water in oil emulsion with a minimum of solid fat phase. These structures are obtained by specific combinations of fat phase characteristics and margarine crystallisation techniques;

- the production of trans-free fat phases by combination of fractionation and/or interesterification on non-hydrogenated and/or fully hydrogenated feedstocks.

\section{Fractionation}

Fractionation is the controlled crystallisation of the "solid" (more saturated) triacylglycerols in a fat mixture followed by separation of the solid and liquid fractions. The main oil, fractionated worldwide, is palm oil, the main reason being the demand for clear liquid oil (olein). More recently, for production of cocoa butter equivalents, cocoa butter replacers and solid fat phases for margarine, there has been a growing interest in the solid product of fractionation (stearin). For stearin production, it only makes sense to fractionate oils that contain relative high solids levels in the 
temperature range of $10-40^{\circ} \mathrm{C}$. The solid phase lines of fat phases produced by fractionation are determined by:

- the type and quality of the feedstock;

- the crystallisation temperature;

- the efficiency of the separation process.

\section{Interesterification}

Interesterification permits a rearrangement or redistribution of the fatty acid chains on the glycerol fragment of the triacylglycerol molecule. Interesterification is promoted by an alkaline catalyst or by lipases (enzymes). Alkaline catalysed reactions produce a mixture of triacylglycerols where the fatty acids are distributed randomly among the fat molecules and randomly among the three available positions within each molecule. Lipases only catalyse rearrangement at the 1 and 3 positions of the glycerol, leaving the ester-link at the 2 position intact.

The modification of the fatty acids distribution will in general lead to an alternation of the solid phase line and the crystallisation behaviour. This is illustrated by two examples:

- random interesterification of a mixture of $50 \%$ tristearate and $50 \%$ trioleate will result in a mixture of six possible triacylglycerols (figure 4). The original binary mixture has a melting point of $65 \circ \mathrm{C}$ and a solids level at $600^{\circ} \mathrm{C}$ of $46 \%$. The melting point of the interesterified six components mixture has decreased to $50 \circ \mathrm{C}$ while the solids level at $60 \circ \mathrm{C}$ dropped to only $5.5 \%$;

- random interesterification of a mixture of two fully hardened components. Figure 5 shows the solid phase lines of the two separate components, and the mixture of the two components before and after interesterification. This shows clearly that the solids levels at high temperature are reduced by interesterification making the solids phase line steeper and the product better suited as fat phase component for margarines than before interesterification.

Trans-free fat phase components

The techniques of interesterification and/or fractionation of a wide variety of different feedstocks may now produce trans-free margarine fat phase components with a range of melting performances.

Liquid seed oils are first fully hydrogenated to generate solids, while the trans level remains very low $(<1 \%)$. They may subsequently be interesterified with non-hydrogenated liquid oil to reduce the solids level at high temperature. This solids level can be further reduced by fractionation (figure 6).

The presence of relative high solids levels in tropical oils creates more flexibility in processing routes.

Fractionation alone will produce a relatively soft stearin component which is not optimal for structuring margarine. 
Fractionation followed by interesterification is used to produce non-hydrogenated fat phase components.

Full hydrogenation followed by interesterification is an alternative to obtain optimal components without fractionation.

This combination of techniques creates a tool for optimal solid fat components production, which is almost as flexible as partial hydrogenation.

The industrial project

The transfer from margarine production based on partially hydrogenated fats to production with very low trans components has been one of the major technical projects in the edible oils and fats business of Unilever in the last decade. It involved:

- development of new fat phase compositions for more than 600 margarine blends with safeguarding of all margarine characteristics;

- optimisation of margarine crystallisation techniques;

- investment in interesterification and fractionation and divestment in hydrogenation;

- a combined exercise of oil buying, production planning and oil/fat stock management to effectively change from the old compositions to the new ones in a short period.

\section{REFERENCES}

1. MENSINK RP, KATAN MB (1990). Effects of dietary trans fatty acids on high-density and lowdensity lipoprotein cholesterol levels in healthy subjects. N Engl J Med, 323: 439-45.

2. WILLETT WC, STAMPFER MJ, et al. (1993). Intakes of trans fatty acids and risk of coronary hearth disease among women. Lancet, 341: 581-5.

3. ASCHERIO A, KATAN MB, et al. (1999). Trans fatty acids and coronary heart disease. N Engl J Med, 340: 1994-8. 
Illustrations

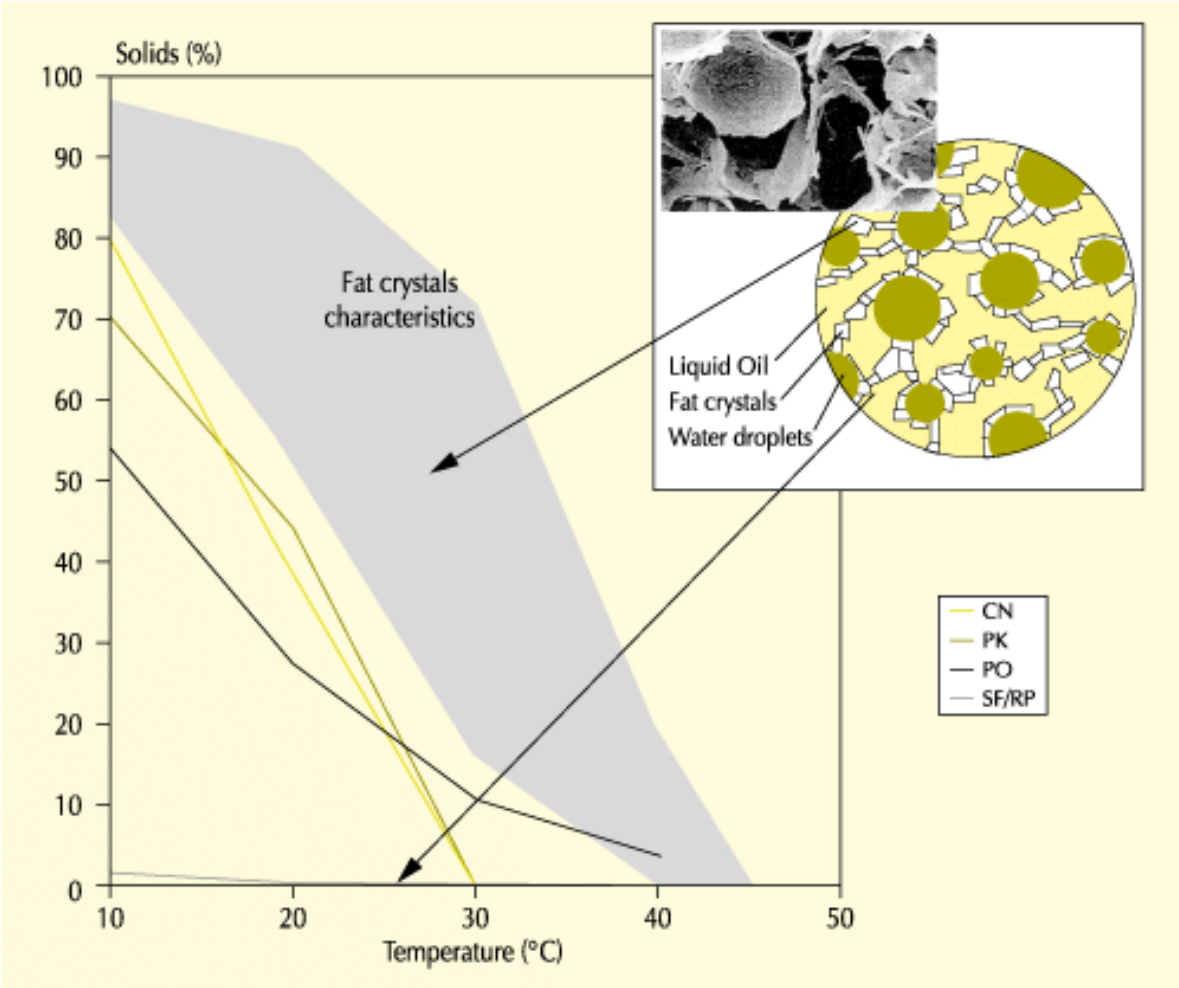

Figure 1. The production of margarines with optimised properties requires the availability of fats with a wide range of solid phase lines (grey coloured area), the oils and fats occurring in nature are too soft for most of these applications - see solid phase lines lines of Coconut (CN), Palmkernel (PK) and Palm Oil (PO). 


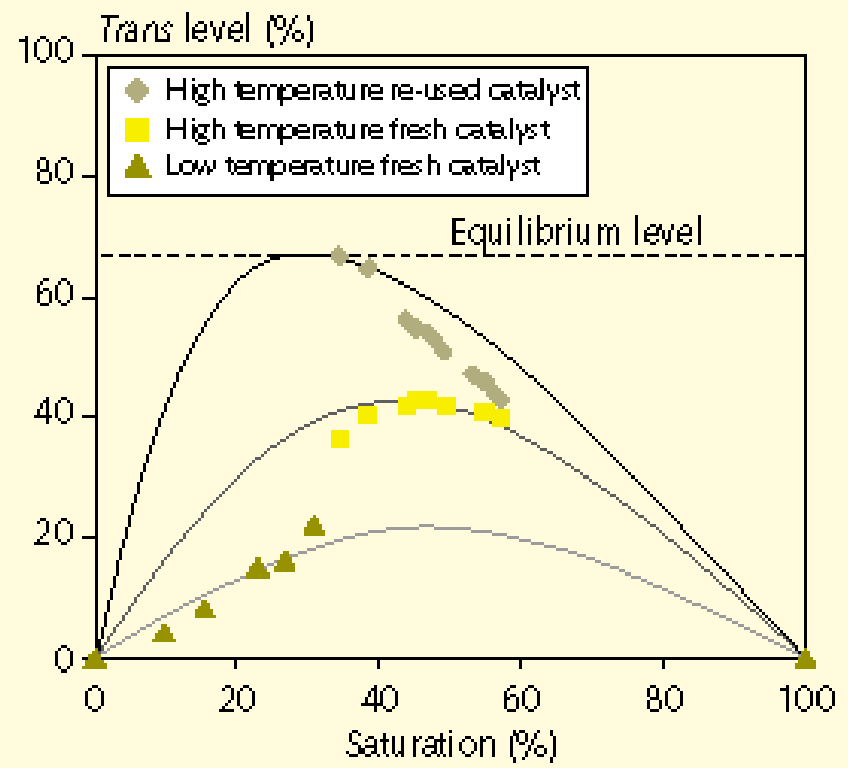

Figure 2. The level of Trans Fatty Acids as function of the degree of saturation. The curve with the highest trans levels is obtained by applying a high hydrogenation temperature $\left(>180^{\circ} \mathrm{C}\right)$, a re-used (sulphur poisoned) catalyst and a low hydrogen pressure. Lower level curves are obtained by reducing the temperature, using a fresh (non-sulphur poisoned) catalyst and increasing the hydrogen pressure.

The points in this graph are trans levels of products, which were used before the introduction of the trans-free policy in 1995. 


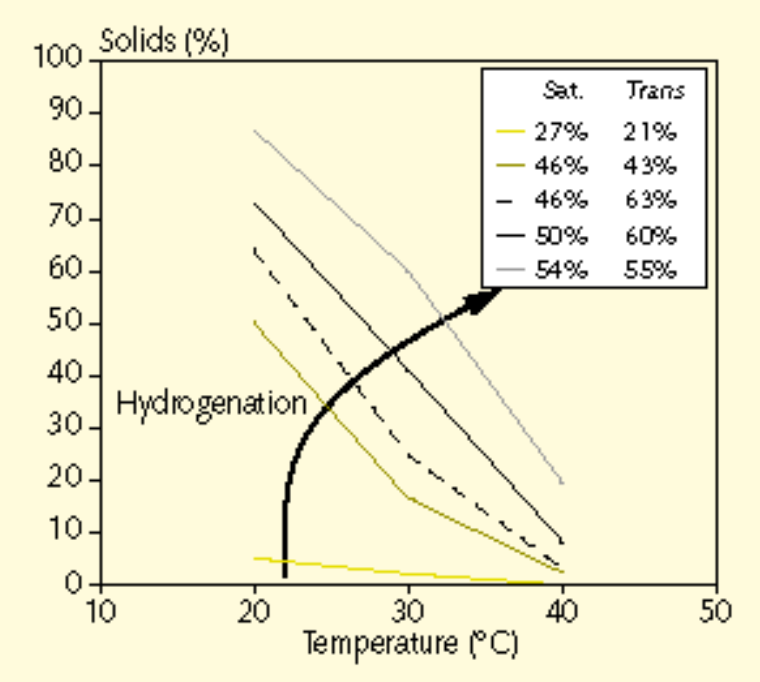

Figure 3. Solid phase lines of hydrogenated Soybean Oil at different degrees of saturation. The two lines at $46 \%$ saturation show a steeper solid line for the product with the higher trans level.

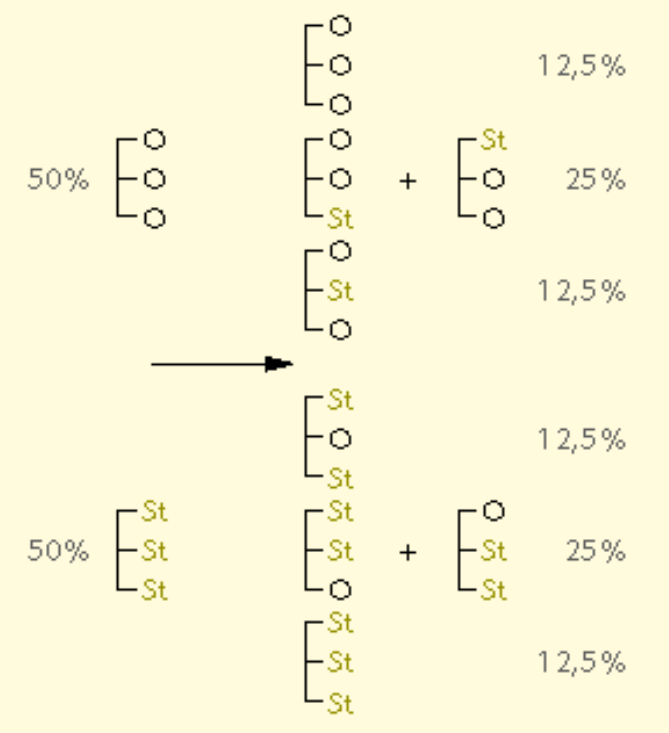

Melting point: $65^{\circ} \mathrm{C}$ $\mathrm{N} 60: 46 \%$

Figure 4. Principles of interesterification. The random distribution of Oleic and Stearic acid in the triacyglycerols after interesterification of 50\% Trioleate and $50 \%$ of Tristearate. 


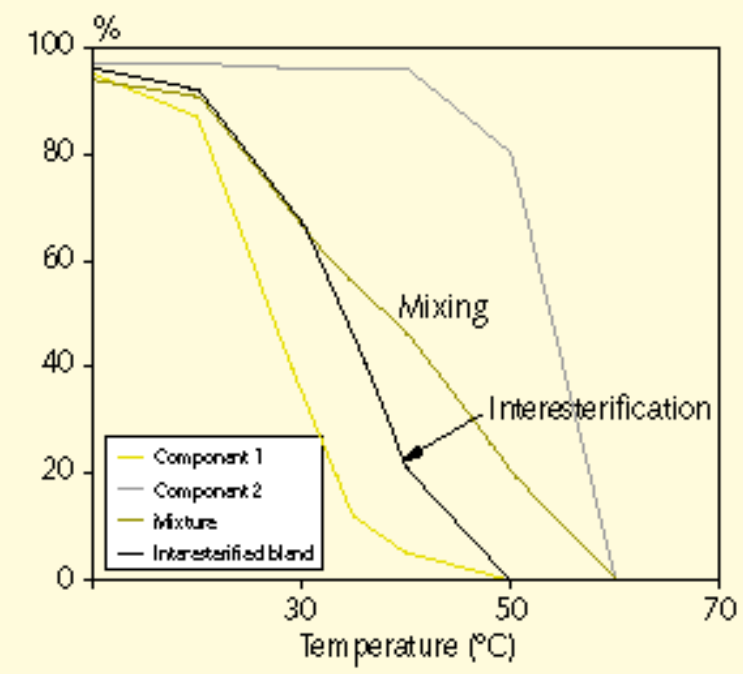

Figure 5. The effect of interesterification on the solid phase lines. Two components are mixed and then interesterified. The solid levels at high temperature decrease due to interesterification while the solid levels at low temperatures remain constant. The steepness of the solid phase line of the product is improved (this effect will not always occur in this way, it depends on the fatty acid compositions of the components of the interesterified mixture).

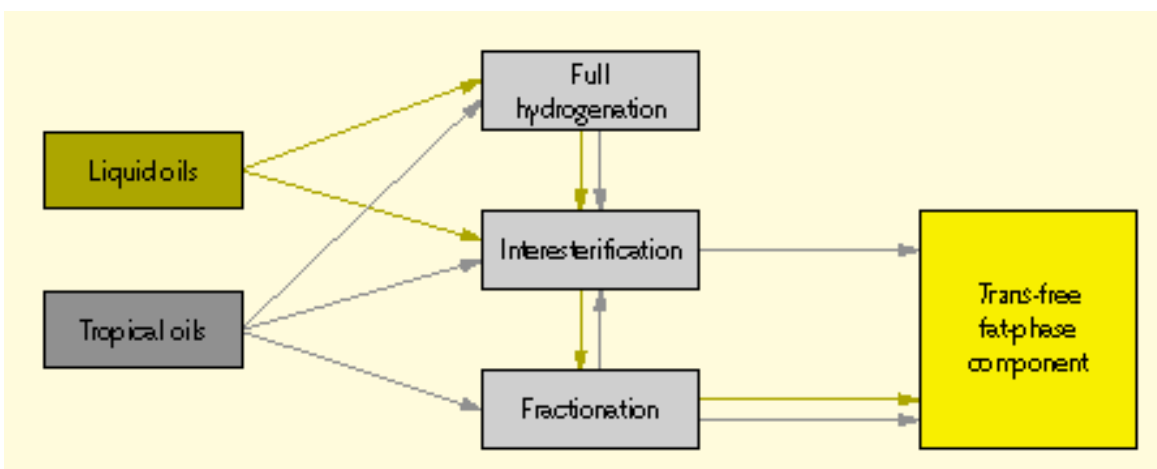

Figure 6. The production of zero trans fat-phase components with a range of melting performances from liquid oils and tropical oils is possible by combination of full hydrogenation, interesterification, and fractionation. 